\title{
STRUCTURE AND CONTROL PRINCIPLE OF A REMOTE-CONTROL SYSTEM FOR MOBILE TERMINAL ELECTROMECHANICAL EQUIPMENT USING AN ANDROID PLATFORM
}

\author{
$\mathrm{Li} W u$ \\ Wuxi Vocational College of Science and Technology, Wuxi 214028, China \\ Email:liwuwvcst@yeah.net
}

\begin{abstract}
In this paper, the structure and control principle of the remote-control system of mobile terminal electromechanical equipment equipped with an Android platform was explored. In the remote control of the intelligent system, the requirement for the mobile platform is relatively high. Therefore, a new mobile remote-control system is selected, and it is mainly characterized by using the Android platform as the terminal and wireless communication technology to control remote electromechanical devices; a mathematical model for DC (Direct Current) motor is established, and Matlab simulation is conducted to design PID (Proportional Integral Derivative) of the remotecontrol system; finally, the system output data are submitted to the host computer. If the data are wrong, the mobile terminal is established to perform real-time monitoring of the equipment. The internal components of the remote monitoring system include server, client, device information transmission module and motion control module. Subsequently, the function of the system is elaborated in detail, by connecting the Android mobile intelligent terminal client with remote electromechanical devices. The analysis shows that the design of the control system is reasonable and the Android mobile intelligent terminal successfully receives the video, which can be played smoothly. Besides, it can stably upload the data of various electromechanical devices, and control them anytime and anywhere, solving the problems of the wide distribution of electromechanical devices and difficult monitoring. The system is simple in operation, low in cost, and easy to assemble. In future research, a more efficient DC motor can be selected to improve the efficiency of the control system.
\end{abstract}

Keywords: Android platform; PID controller; Remote monitoring system; Matlab.

\section{Introduction}

Since the 1970s, factories attach importance to technology development for higher economic profits. In this process, the workers must be very familiar with the use of mechanized equipment. Meantime, the equipment is updated very quickly, and the introduction speed of equipment is also very fast. In particular, some of the top large domestic manufacturers introduce the most advanced production equipment [1]. However, after the introduction of too much new equipment, there is a lack of management and maintenance of the equipment, and there are many problems in the supervision, resulting in a lot of unexpected losses. The losses mainly include the following three aspects: (1) with the development of science and technology, many factories invest a lot in equipment. This causes management problems, which not only lead to low production efficiency, but also waste a lot of equipment resources, leading to large investment and production cost; (2) manual operation supervision or regular manual inspection are still used. As a result, the work efficiency cannot be improved, and the timeliness is poor; (3) although the advanced production equipment is introduced, the supervision quality of equipment management personnel is low. For example, some supervisors are hired temporarily to work in the factory, and do not understand the basic status of the factory; and some technicians are just starting to work and lack experience in equipment management and maintenance [2].

In recent years, robots are often sent to various working environments to conduct detection and exploration for improving their ability of remote control. Because of its strong adaptability, the robot plays an increasingly important role in various fields. The remote control of the robot can be realized via the network, which can further expand the working range, especially in the fields of remote medical assistance and equipment sharing [3]. The remote control is realized by the remote devices based on wireless network signals. 
The remote control is usually carried out on the network, and it generally adopts two kinds of program control: the client and the server. The client is installed on the controller's computer, while the server is installed in the mechanical equipment and controlled by the signal generated by the client. Generally, the basic principle of industrial remote intelligent control and service is that the operator sends the control instructions to the near-end controller at the far end of the site by using the remote-control equipment, and then the near-end controller completes the control to achieve the equipment monitoring [4]. The monitoring system returns the results of the control and the state of the controlled equipment to the cloud computing processing centre to complete intelligent control and service. The remote-control technology is concentrated by automation and remote control, that is, when the remote control of mechanical equipment is realized, the automation of machinery is also achieved. This achieves intelligent mechanical control, reduces the risk of operation, improves the personal safety of technical personnel, and promotes social progress and national influence [5].

In terms of the current technology, the remote control of most robot controls is conducted by using a PC (Personal Computer), GSMC/GPRS (Global System for Mobile Communications/General Packet Radio Service), or PC of WiFi (Wireless Fidelity) to control H1, etc. These control methods still rely on PC terminals, and a specific workplace and exclusive network to control the scene need to be set, which indicates that the traditional method has many disadvantages [6]. With the rapid development of electronic information technology, the mobile intelligent terminal gradually replaces the PC terminal because of its strong advantages. The running speed of the mobile intelligent terminal is as several times as that of the PC, which can not only realize the remote control of the robot, but also achieve the effect of real-time supervision. Nowadays, in the intelligent terminal market, the Android system's superior performance and external system compatibility win more and more shares in the market. Therefore, the Android system becomes the preferred target in doing related research. Since the Android system is powerful, it can be used as the platform of the remote-control system to support the required algorithm in the remote-control system of electromechanical equipment. In the control system, a WiFi network is used to connect the Android terminal TCP/IP (Transmission Control Protocol/Internet Protocol), thereby connecting with the mobile robot to realize the remote control and monitor the mechanical and electrical equipment $[7,8]$.

The establishment of a mobile terminal equipped with an Android platform realizes the remote monitoring system, solves the inconvenience of traditional wired monitoring mode, and provides a more reliable and convenient scheme for the monitoring of intelligent battery, which has practical significance.

\section{Design and Method of Control System 2.1 Design of Driving Circuit}

When a two-phase outer rotor is controlled by a brushless DC (Direct Current) motor, half-bridge control is often used. The fan products of the PAPST MULTI FAN series, a famous foreign brand, are taken as the object. This kind of fan belongs to the threewire fan, and there are three wires among them. Each wire represents different meanings. They are positive wire of power supply, ground wire of power supply and wire for detecting motor speed. However, it is found that the efficiency of the motor coil controlled in this way is not high. Figure 1 is the working principle diagram of the motor. In the figure, there are two coils in the motor, which are controlled by two Darlington tubes. According to the received Hall sensor signals, the on command and the off command are made respectively. Since the motor pole shoe has the property of asymmetry, the starting torque is obtained [9].

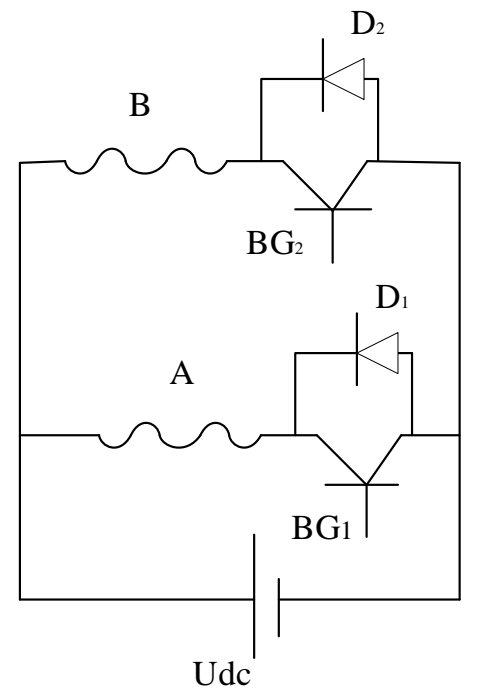

Figure 1: Working principle of DC motor

The controller design adopts the way of a fullbridge drive, which reversely parallels the A phase and $B$ phase of the coil. In this case, when the pole shoe is electrified, it will produce an opposite magnetic field. The main feature of the full-bridge power inverter bridge is that the bridge is controlled independently by an $\mathrm{H}$-bridge, which is flexible and easy to control the magnitude and direction of the variable winding current [10]. In addition, the interlocking design mode is adopted for the control system to prevent the power converters of the upper bridge and lower bridge from a direct short circuit. 
In Figure 2, there are five wires in the drive circuit, and they are Vcc, G, speed detection line, the input line of PWM (Pulse Width Module), and current detection line. The power supply in the circuit is an active device with and without a gate. $\mathrm{Vcc}$ is the power supply with a rated voltage of $12 \mathrm{~V}$ (allowable change of positive and negative 21\%), and the maximum allowable current of $4 \mathrm{~A}$.

\subsection{Establishment of Mathematical Model and Relevant Control Algorithm}

\section{A. Establishment of the mathematical model of motor \\ In the normal case, the winding voltage balance} equation of a two-phase Brushless DC motor is:

$$
\left[\begin{array}{l}
U_{A} \\
U_{B}
\end{array}\right]=\left[\begin{array}{ll}
R & O \\
O & \mathrm{R}
\end{array}\right]\left[\begin{array}{l}
i_{A} \\
i_{B}
\end{array}\right]+\left[\begin{array}{ll}
L-M & 0 \\
0 & \mathrm{~L}-\mathrm{M}
\end{array}\right] \frac{d}{d t}\left[\begin{array}{l}
i_{A} \\
i_{B}
\end{array}\right]+\left[\begin{array}{l}
e_{A} \\
e_{B}
\end{array}\right]
$$

In the above equation, $U_{A}$ and $U_{B}$ are phase voltage of stator winding; $\mathrm{R}$ is resistance; $i_{A}$ and $i_{B}$ are winding current; $e_{A}$ and $e_{B}$ are the electromotive force of stator winding; $L$ is self-induction, and $M$ is mutual inductance of phase winding.

The equation of voltage balance is deduced by calculating equation (1):

$$
u=r^{\prime} i+L^{\prime} \frac{d i}{d t}+k_{e} \omega
$$

In equation (2), $u$ is the voltage at both ends of the motor; $i$ is the phase current, $r^{\prime}$ is the resistance; $L^{\prime}$ is the phase inductance; $K_{e}$ is the back EMF (Electronic Manufacturing Facility) coefficient, and $\omega$ is the angular velocity of the motor. The corresponding torque balance equation of the BLDC (Brushless Direct Current) motor is as follows:

$$
\begin{aligned}
& T_{e}-T_{l}=J \frac{d \omega}{d t}+P \omega \\
& T_{e}=k_{t} i
\end{aligned}
$$

In the above equations, $T_{e}$ is the electromagnetic torque; $T_{1}$ is the load torque; $J$ is the moment of inertia; $K_{t}$ is the torque coefficient, and $P$ is the damping coefficient. From equations (2-4), it can be concluded that the motor can be represented by a second-order system. The system transmission function is shown in equation (5), and the corresponding system block diagram is shown in Figure 2:

$$
\frac{\omega(\mathrm{s})}{U(\mathrm{~s})}=\frac{K_{t}}{\left(\mathrm{~L}^{\prime} \mathrm{s}+\mathrm{r}^{\prime}\right)(\mathrm{J} \mathrm{s}+\mathrm{P})+\mathrm{K}_{\mathrm{t}} \mathrm{K}_{\mathrm{e}}}
$$

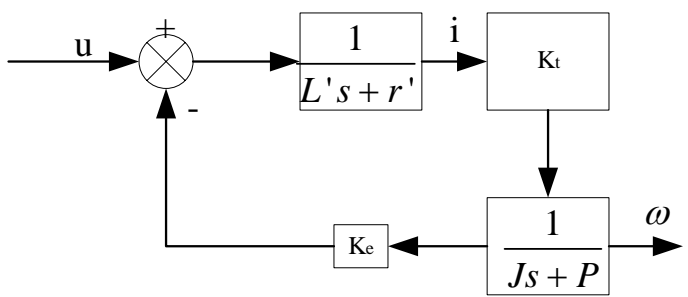

Figure 2: DC motor control system

\section{B. Motor drive and control}

In the control system, the 16-bit microcontroller is selected as the controller, and the PWM data signal drives the MOSFET (Metal Oxide Semiconductor Field Effect Transistor), expanding the speed range of the whole control system and improving the accuracy. The transistor is used as a switch to control the power supply, and a fixed frequency parameter is set to turn on and off the power supply. According to the needs of the experiment, the time of turning on and off the power supply in the whole cycle is adjusted by changing the "duty cycle" of the voltage on the armature of the BLDC motor. In this way, the motor speed can be adjusted in real-time [11]. This control method is mainly suitable for some small and medium power motors. In the design of a remote-control system for electromechanical equipment, the voltage loop single closed-loop control mode is adopted due to the economic factor. PID (Proportional Integral Derivative) control algorithm is adopted in the system, and the proportion of PWM signal is changed through equation calculation to adjust the motor speed in the system.

Based on the above system, the PID control algorithm can be divided into five types:

(1) When $\mathrm{e}(k) \geq M$, the deviation is very great, and the maximum value of the controller is the output:

(2) When e $(k) \Delta e(k)>0$ or $\Delta \mathrm{e}(\mathrm{k})=0$, it indicates that the actual value is more and more different from the set value.

$$
u(\mathrm{k})\left\{\begin{array}{ll}
u(\mathrm{k}-1)+2.0 \mathrm{~K}_{\mathrm{p}} \mathrm{e}(\mathrm{k}) & \mathrm{e}(\mathrm{K}) \geq M_{2} \\
u(\mathrm{k}-1)+1.5 \mathrm{~K}_{\mathrm{p}} \mathrm{e}(\mathrm{k}) & \mathrm{e}(\mathrm{K})<M_{2}
\end{array}\right\}
$$

(3) When $e(\mathrm{k}) \Delta \mathrm{e}(\mathrm{k})<0 \quad$ and $\Delta e(\mathrm{k}-1) \Delta \mathrm{e}(\mathrm{k})<0$ are achieved, or $\mathrm{e}(\mathrm{k})=0$, it shows that the actual value is near the set value or the set value is reached.

$$
u(\mathrm{k})=\mathrm{u}(\mathrm{k}-1)
$$

$$
\text { (4) When } e(\mathrm{k}) \Delta \mathrm{e}(\mathrm{k})<0
$$

$\Delta e(\mathrm{k}-1) \Delta \mathrm{e}(\mathrm{k})<0$ are achieved, and the error is in the extreme state, the actual value is oscillating up and down 


$$
u(\mathrm{k})\left\{\begin{array}{ll}
u(\mathrm{k}-1)+2.0 \mathrm{~K}_{\mathrm{p}} \mathrm{e}(\mathrm{k}) & \mathrm{e}(\mathrm{K}) \geq M_{3} \\
u(\mathrm{k}-1)+1.5 \mathrm{~K}_{\mathrm{p}} \mathrm{e}(\mathrm{k}) & \mathrm{e}(\mathrm{K})<M_{3}
\end{array}\right\}
$$

(5) When e $(\mathrm{k}) \leqq \mathrm{M} 4$, and the deviation is very small, it is controlled by ordinary PID.

$$
u(\mathrm{k})=\mathrm{Kp}_{\mathrm{e}}(\mathrm{k})+0.5 \mathrm{k}_{\mathrm{I}} \sum_{i}^{k} e(\mathrm{i})+\mathrm{K}_{\mathrm{D}}[\mathrm{e}(\mathrm{k})-\mathrm{e}(\mathrm{k}-1)]
$$

In equation (9), M1, M2, M3, and M4 are the thresholds respectively.

\subsection{System Design}

Android system is an open-source system of Linux, which is developed by Google in the United States. This system includes an operating system, user control interface, and various application software, and there is no exclusive right barrier like other systems, which hinders the innovation of the industry. The object-oriented programming language used in the Android system is completely open [12].

Therefore, the system is mainly designed through the client/server framework to connect the server and client of remote electromechanical equipment. The control system principle of the remote device is that the server sends the real-time device condition monitored by the camera to the Android client, and the client receives the specific information of the device and sends real-time commands to the control system through video observation.

\section{A. Implementation of video transmission}

In the system, the connection between the client and the device port is through the transmission control/Internet communication protocol for data exchange. The server is based on Java Media Framework, which is a newly developed application program interface and can playback data and exchange real-time data [13].

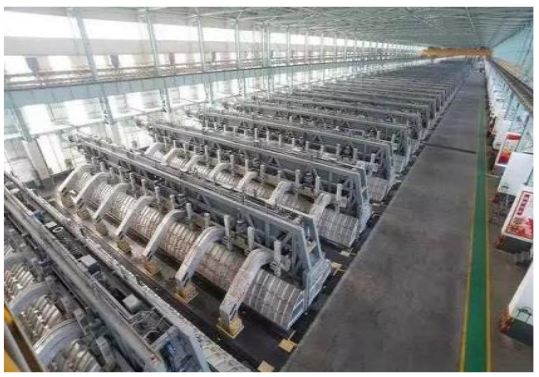

(a)
Figure 3 shows the server-side interface of video data acquisition system of mobile robot camera based on JMF:

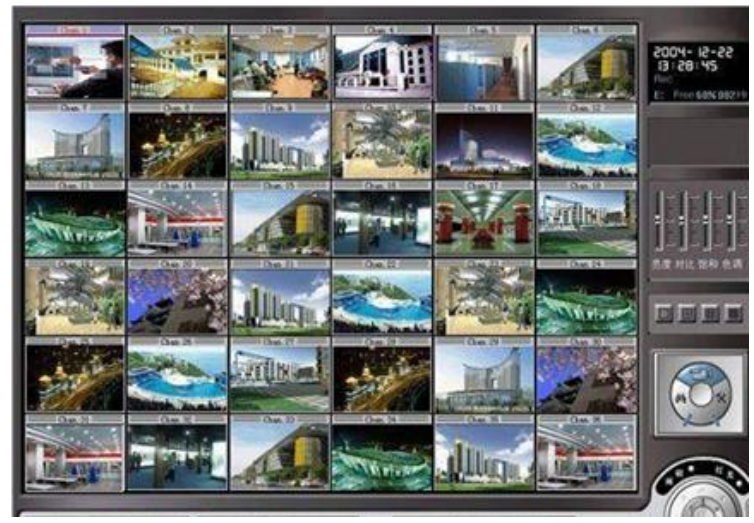

Figure 3: Server monitoring screen

The design planning type of data transmission involves ImageGrabber and VideoSencer. The realtime working videos of the device are obtained through Java Media Framework. After the server of the control system is started, the ImageGrabber starts a thread, which is responsible for saving the fieldwork video data captured by the camera locally. In this case, the server is waiting for the client to connect. When the client connects to the server, the server starts a new thread to access the video data of the client browser. Sometimes, when the server sends the information of a video to the client, it can send about 8 videos in a second [14].

The client is composed of Activity: MainActivity and Con: Trolleractivity. When the user logs in, a page appears. After the IP and port number of the corresponding server are entered, the user can connect with the server through a socket. At the moment, the client creates a new thread for the image data sent by the server, and the client maps one image at a time and displays the video in the user's interface. Figure 4 shows the real-time videos received by the client:

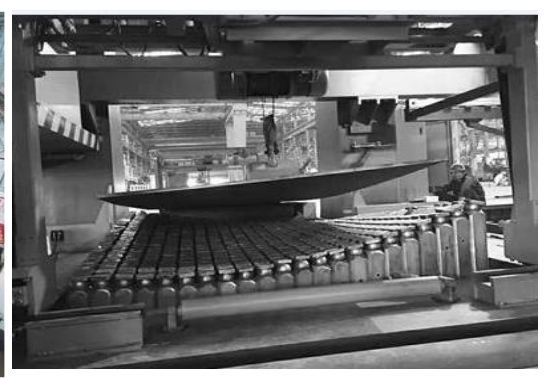

(b)

Figure 4: Monitoring client interface (a: plant condition; $b$ : machine internal condition)

\section{B. Realization of remote control}

In the whole control system, the remote-control module and the scene video module are not together, and different ports are used to connect the server.
AS-R (Acceleration Slip Regulation) mobile robot has a simple composition, which is mainly controlled by two separate motors. Therefore, a turn of AS-R is generated by the wheel speed difference of two 
driving wheels in motion. Because of the characteristics of the robot, the most direct and effective way to control the robot is to use the system. In the system, two driving wheels adjust the speed of the servo motor and finally complete the task.

$v$ is the maximum speed that the robot can reach, $v l$ is the left wheel speed, and the speed of the right wheel is $v r$. When the hand contact is at $c$, $v_{1}=v \times(\mathrm{r} / \mathrm{R})$ and $v_{r}=v \times(\mathrm{r} / \mathrm{R}) \bullet \cos (\alpha)$ are achieved. The above equations should meet the requirements: $0<a<90^{\circ}$; $\cos (a)<1$, and $v l>v r$. In this case, the robot moves to the right; when the hand contact is reaching $a$, the conditions, $a=0^{\circ}, \cos$ (a) $=1$, and $v l=v r$, should be met, and the robot goes straight. When the robot reaches $b, a=90^{\circ}, \cos (a)=$ 0 , and $v r=0$ should be met, and the range of the robot moving to the right is the least. After the analysis, two conclusions are drawn.

(1) If $r$ increases, the wheel speed of the relative robot will also increase;

(2) The larger $a$ is, the larger the wheel speed ratio on the left and right of the robot is, that is, the smaller the turning radius of the robot wheel is, the larger the turning range of the robot is.

The control communication protocol of the system is saved through the control command interface. The client control command interface is successfully connected to the server. When the client enters an account, it will log in successfully. And a new thread is started and the BUILD_SYSTEM signal is sent to the server. When the corresponding server program receives the relevant control signal, it will use JNI (Java nationality interface) to control the robot's movements, obtaining a robot control case [15].

\section{Communication design}

After the completion of the hardware and model design, the data transmission protocol is studied to collect the data of PLC (Programmable Logic Controller) and other devices based on the discussion with the mechanical engineer [16]. The data structure of the remote device is used to send the request to the remote device based on the TCP protocol. And then the back-end sends the obtained data to the front-end, and the terminal processes the received data information [17].

In terms of the data of construction machinery equipment, a data transmission protocol is established for the client by using the bus of the mechanical and electrical equipment. The information on mechanical and electrical equipment needs to be subcontracted. The size of an information packet is 8 bytes, and each actuator contains multiple packets. The information packet contains all the information that the mechanical and electrical equipment needs to transmit.

D. Design of software in the system

In the remote device control system, it is necessary to determine the hardware structure module, the working mode of the device, the transmission protocol of the client and server, and the design of the software. Besides, the software design is also the focus that needs to be discussed, because the design of the software not only affects the underlying hardware, but also determines the working mode of the terminal according to the specific working conditions of the remote electromechanical equipment. Moreover, it realizes ULTRA LONG-distance real-time monitoring of mechanical and electrical equipment [18]. Generally, remote electromechanical equipment has a highspeed and good positioning function. The CPU is ARM Cortex - M3 series microprocessor LPC1778, for it has many advantages, like high integration and simple structure. The $\mu \mathrm{COS}$ - II operating system is used to develop the program because it has such functions as kernel initialization, task processing, time processing, task synchronization, communication, and CPU porting. And it also supports cortex-m3/M1/M and other kernel processors. The developed $\mu \mathrm{COS}$ - II is used to initialize the hardware system and the operating system, and create initial tasks and process multiple tasks $[19,20]$.

\section{Simulation Analysis 3.1 Analysis of Matlab Simulation Results}

The $\mathrm{M}$ file of Matlab is used for programming. The parameters of the controlled motor are shown in Table 1.

Table 1 Motor parameters

\begin{tabular}{|c|c|c|c|c|c|c|}
\hline \multirow{4}{*}{ 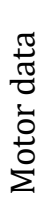 } & $V_{\text {rated }}$ & Irated & NM & $\mathrm{R}$ & $\mathrm{P}$ & Friction mode \\
\hline & 11 & 3.6 & 2 & 0.5 & 1 & Rolling friction \\
\hline & PI & $\mathrm{J}$ & $\mathrm{Z}$ & \multicolumn{2}{|c|}{$E_{\text {back }}$} & $\mathrm{M}$ \\
\hline & 0.1 & $1.5^{*} 10^{-6}$ & $1 * 10^{-5}$ & \multicolumn{2}{|c|}{0.125} & 0.034 \\
\hline
\end{tabular}

(PI: phase inductance; J: the moment of inertia; Z: damping coefficient; E: back EMF; M: torque; NM: number of phases of the motor; P: number of poles)

Matlab simulation results are shown in Figure 5. Figure (a) is the speed output comparison curve of of the controllers. the system. Figure (b) shows the output comparison 

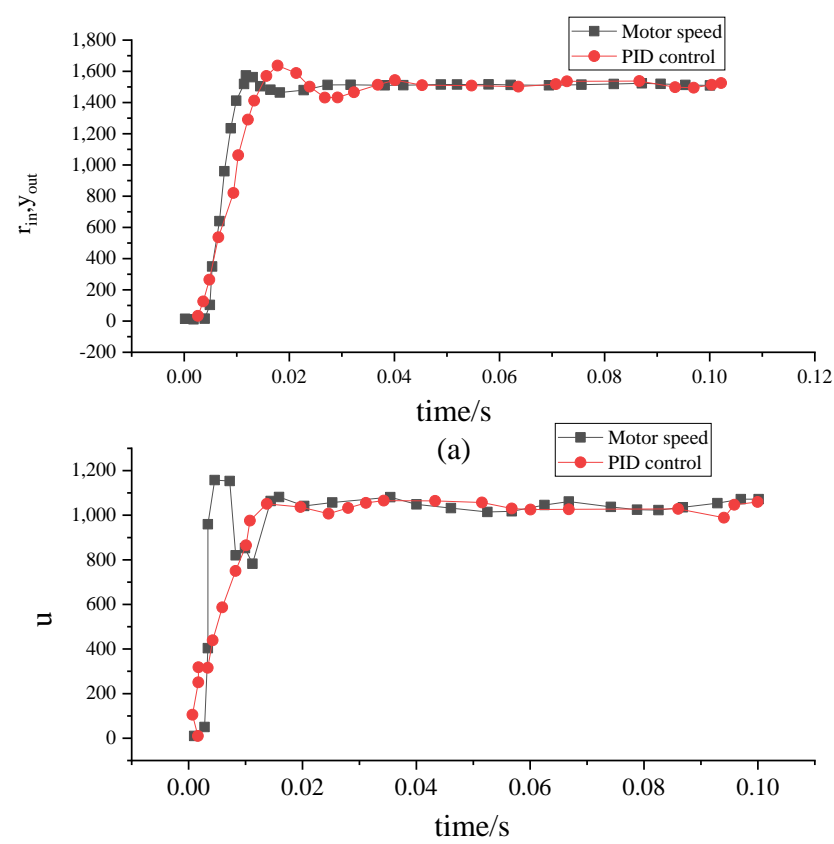

(b)

Figure 5: Control line curve (a: system output curve; b: controller output curve)

The PID control system has a faster response speed, and the signal can be output stably after a vibration, which is shown in Figure (a).

The response of ordinary PID is slower than expert PID, and the system needs to vibrate about 3 times to achieve relative stability.

In Figure (b), the expert PID control can output the extreme value of the controller directly if there is a large deviation, which increases the response speed of the system.

\subsection{Analysis of the Measured Data}

The above algorithms are transplanted to a 16-bit FreescalesXS128 single-chip microcomputer to control the motor.

The motor is DC motor PEB0812DHE; IRF9530 and IRF530 are the MOSFET of the H-bridge control circuit, and the hall element model is U18.

PID controls the remote electromechanical equipment and uploads the real-time data of the motor to the host computer by data exchange. The data transmission frequency is $20 \mathrm{~Hz}$. Figure 6 below shows the measured information:
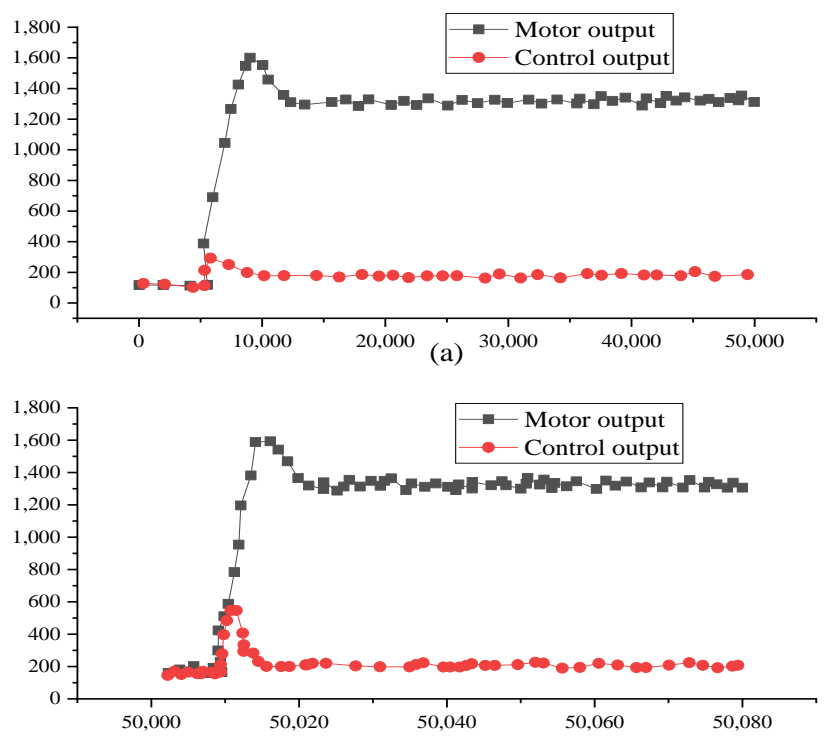

(b)

Figure 6: Corresponding curve of PID control (a: ordinary PID control; b: expert PID control) 
The measured data show that the output response of the controlled object is faster and the regulation times are less under the expert PID control.

\section{Discussion}

In the research of electromechanical equipment control system, many experiments and tests are carried out, including the expected index test according to the research standard, the test of adding pressure to a single function, the single function pressure test, and the test in various complex working environments [21]. The design of hardware and terminal software in the control system meets the expected requirements of the research. And it can also meet the real-time supervision and control of electromechanical equipment. Therefore, the intelligent monitoring system based on the Android platform can effectively monitor the devices in different environments simultaneously, and increase or decrease the controlled devices according to the actual needs. It can also select sensors and feedback facilities properly to reduce production costs and achieve high efficiency and high practicability [22]. In the process of monitoring, it is expected that restricting data are used to transfer bytes, which helps the software terminal execute the command issued by the monitor more accurately. To realize the purpose, the STM32F4 with $168 \mathrm{MHz}$, fast judgment speed and high main frequency, is selected as the main control to monitor and control the equipment in safe production [23].

\section{Conclusion}

According to the actual needs of remote control, a DC motor control system is designed. The DC motor is first analysed, and then the corresponding model is constructed. Furthermore, the advantages of the expert PID are demonstrated in accordance with the best actual parameters of the system, and PID simulation based on Matlab is conducted. The analysis of the measured data proves that the designed control system has faster response speed, higher stability and anti-interference ability. Besides, it is a very effective way to realize the remote control of mechanical and electrical equipment using the Android platform.

In the information age, the system makes full use of the development advantages of the Internet of things platform with Android as the main body, to realize the unified monitoring of different devices in different working environments with the help of each module of wireless data. Since the distribution of mechanical and electrical equipment is relatively large and scattered, they are difficult to be monitored and controlled. Therefore, the new working model of remote monitoring of mechanical and electrical equipment is proposed. After the hardware and software are designed, the data can be transferred to achieve monitoring. The remotecontrol system can stably upload various types of electromechanical equipment data, and control the operation of the equipment anytime and anywhere, which solves the problem of wide distribution and difficult monitoring of electromechanical equipment. The system is simple in operation, low in cost and easy to install, and deserves to be widely used. However, there are some shortcomings, which have to be improved. The stability and control experience of the remote-control system based on Android platform need to be studied further.

\section{References}

[1] Jia Y. J. (2021) Design of an Intelligent Greenhouse Remote Control System Based on a Fuzzy Neural Network. ijat, 15(2), 243-248.

[2] Wang M., Zhang B. H. (2021) Analysis of Internet of Things Computer Network Security and Remote Control Technology. IOP Conference Series: Earth and Environmental Science, 634(1), 012035.

[3] Dudnikov S. Y., Vertegel V. V., Golovin V V, et al. (2021) Remote control system for the unmanned floating platform. IOP Conference Series: Materials Science and Engineering, 1047(1), 012130.

[4] Syafruddin R., Ramady G. D., Herawati Y. S, et al. (2021) Remote Control Target Tracking Using GPS / INS-IMU. Journal of Physics: Conference Series, 1783(1), 012044.

[5] Lin Y. N., Wang S. K., Yang C. Y, et al. (2020) Development and verification of a smart remote control system for home appliances. Computers \& Electrical Engineering, 88, 106889.

[6] Uljayev E., Ravutov S. T., Ubaydullayev U. M. (2020) Remote control device to control the contact uniformity of the brush strippers on the spindle's surface of the cotton picking apparatus. IOP Conference Series: Earth and Environmental Science, 614(1), 012139.

[7] Sangmo K. (2020) Advanced MicroActuator/Robot Fabrication Using Ultrafast Laser Direct Writing and Its Remote Control. Applied Sciences, 10(23), 8563-8563.

[8] Gravano D. M., Chakraborty U., Pesce I, et al. (2020) Solutions for Shared Resource Lab Remote Quality Control and Instrument Troubleshooting during a Pandemic. Cytometry Part A, 99(1), 51-59.

[9] Garzotto F., Comoretto R. I., Ostermann M, et al. (2021) Preventing infectious diseases in Intensive Care Unit by medical devices remote control: Lessons from COVID-19. Journal of Critical Care, 61, 119-124. 
[10] Jiao H. W., Ma Z. M., Hua W. R. (2020) The Design of the Control System of Fire Control and Smoke Extraction Robot Based on PLC. Journal of Physics: Conference Series, 1646(1), 012093.

[11] Yu P. M., Kukhtin S. M., Bashchenko S. M, et al. (2018) Principle of remote control over the presence of dissolved gases in water media. Physical Oceanography, 20(4), 308-316.

[12] Agata A., Adam M. (2018) Capabilities of Local and Remote Monitoring and Control of Equipment Used in Marine Ship Industry. New Trends in Production Engineering, 1(1), 135-141.

[13] Zheng X. X., Liu N., Wang W. (2018) New Problems and Improvement Ideas of Medical Equipment Quality Control. Zhongguo yi liao qi xie za zhi $=$ Chinese journal of medical instrumentation, 2018, 42(2), 150-153.

[14] Junmo K., Daegeun H., Damdae P, et al. (2017) Design of optical emission spectroscopy based plasma parameter controller for real-time advanced equipment control. Computers and Chemical Engineering, 100, 38-47.

[15] He L., He L. B., Yuan X, et al. (2021) Beidou-GPS dual mode positioning of mobile communication equipment based on Android platform. Journal of Intelligent \& Fuzzy Systems, 40(2), 2917-2928.

[16] Adersh G. A., Sibi S., Surej K. L. K, et al. (2021) "Canine tracker"; an app based on android platform for localisation of impacted maxillary canines using digital panoramic radigraphs. Journal of Oral Biology and Craniofacial Research, 11(1), 9-12.
[17] Chen S. H., Jiu Z. (2020) A Method of Stereoscopic Display for Dynamic 3D Graphics on Android Platform. Journal of Web Engineering, 19(5), 849-864.

[18] Amirulloh I., Iskandar I. d., Apriyani Y, et al. (2020) Teacher Attendance Monitoring System Teaching with QR-Code and Geo Location using Android Platform. Journal of Physics: Conference Series, 1641(1), 012030.

[19] Yulianto B., Alfarisi S., Nurfaalih N. T. (2020) Free Feeder/Bus Ticketing Systems Modelling for Students in Surakarta City Based on Android Platform. Journal of physics. Conference series, 2020, 1625(1), 012030.

[20] Gökçer P., Mete E., Korhan K. (2020) Feature Selection for Malware Detection on the Android Platform Based on Differences of IDF Values. Journal of Computer Science and Technology, 35(4), 946-962.

[21] Ketut S. I., Suwintana I. K., Sudiadnyani I. O. (2020) The development of information system for garments industry using the android platform. Journal of Physics: Conference Series, 1569(2), 022007.

[22] Manas S., Debabrata M. (2019) crystallographic tools for the Android platform. Journal of Applied Crystallography, 52(6), 1449-1454.

[23] Marwa A. A., Blake A., Stephen J, et al. (2019) The development of an Android platform to undertake a discrete choice experiment in a low resource setting. BioMed Central, 77(1), 20. 